\title{
Active tuberculosis risk associated with malignancies: an 18-year retrospective cohort study in Korea
}

\author{
Jaekyung Cheon ${ }^{1}$, Changyoung Kim ${ }^{2}$, Eun Ji Park ${ }^{3}$, Minsu Ock ${ }^{4}$, Hyeji Lee ${ }^{5}$, Jong Joon Ahn ${ }^{6}$, \\ Yangjin Jegal $^{6}$, Kwang Won Seo ${ }^{6}$, Soon Eun Park ${ }^{7}$, Ilsang Han ${ }^{7}$, Hojun Kang ${ }^{7}$, Mingi An ${ }^{7}$, Jongha Park ${ }^{8}$, \\ Gyung-Min Park ${ }^{9}$, Jae-Bum Jun ${ }^{10 \#}$, Taehoon Lee ${ }^{6 \#}$
}

${ }^{1}$ Division of Hematology-Oncology, Department of Internal Medicine, ${ }^{2}$ Biomedical Research Center, ${ }^{3}$ Medical Information Center, ${ }^{4}$ Department of Preventive Medicine, ${ }^{5}$ Department of Emergency Medicine, ${ }^{6}$ Division of Pulmonary and Critical Care Medicine, Department of Internal Medicine, ${ }^{7}$ Department of Anesthesiology and Pain Medicine, ${ }^{8}$ Division of Nephrology, Department of Internal Medicine, ${ }^{9}$ Division of Cardiology, Department of Internal Medicine, ${ }^{10}$ Division of Infectious Diseases, Department of Internal Medicine, Ulsan University Hospital, University of Ulsan College of Medicine, Ulsan, Korea

Contributions: (I) Conception and design: J Cheon, JB Jun, T Lee; (II) Administrative support: J Park, GM Park; (III) Provision of study materials or patients: C Kim, EJ Park, M Ock; (IV) Collection and assembly of data: All authors; (V) Data analysis and interpretation: All authors; (VI) Manuscript writing: All authors; (VII) Final approval of manuscript: All authors.

"These authors contributed equally to this work.

Correspondence to: Taehoon Lee, MD, PhD. Division of Pulmonary and Critical Care Medicine, Department of Internal Medicine, Ulsan University Hospital, University of Ulsan College of Medicine, 877, Bangojinuinhwando-ro, Dong-gu, Ulsan, 44033, Korea. Email: tleepulalg@uuh.ulsan.kr; Jae-Bum Jun, MD, PhD. Division of Infectious Diseases, Department of Internal Medicine, Ulsan University Hospital, University of Ulsan College of Medicine, 877, Bangojinuinhwando-ro, Dong-gu, Ulsan, 44033, Korea. Email: jjb@uuh.ulsan.kr.

Background: Active tuberculosis (TB) develops in approximately $10 \%$ of people with a latent tuberculosis infection (LTBI). TB guidelines recommend that LTBI screening and treatments target high-risk patients. Malignancies are not universally considered a high-risk factor for active TB. This study aimed to determine the degrees to which active TB risk was associated with various cancers in a Korean population.

Methods: This study involved patients aged $\geq 20$ years who were diagnosed with cancer at Ulsan University Hospital (UUH) from January 2000 to December 2014 and individuals who visited UUH for health screening and were age- and sex-matched randomly with cases in a 1:2 ratio. Using retrospective cohort study, the development of bacteriologically confirmed TB (BCTB) within 3 years after enrollment was investigated. The relative risks of BCTB were estimated using incidence rate ratios (IRRs) and a Poisson regression analysis.

Results: During the study period, 380 of 34,783 cancer patients and 79 of 69,566 control subjects developed BCTB, yielding respective incidence rates of 535 and 37/100,000 person-years, respectively. In all cancer cases, the IRR of BCTB was 14.30, and especially high rates were associated with the following cancers: esophageal cancer (74.72), multiple myeloma (70.76), lung cancer (50.35), pancreatic cancer (46.04), leukemia (40.45), head and neck cancer (24.60), and lymphoma (22.67).

Conclusions: The incidence of active TB was higher in cancer patients than in control subjects. In particular, lung cancer, esophageal cancer, pancreatic cancer, hematologic malignancy and head and neck cancer were identified as high-risk factors for active TB, as indicated by IRRs of 20-75. These findings suggest that patients with high-risk cancers should be targeted for LTBI screening and treatment.

Keywords: Tuberculosis (TB); latent tuberculosis infection (LTBI); Mycobacterium tuberculosis; tuberculosis screening; cancer; malignancy

Submitted Oct 21, 2019. Accepted for publication Feb 12, 2020.

doi: $10.21037 /$ jtd.2020.02.50

View this article at: http://dx.doi.org/10.21037/jtd.2020.02.50 


\section{Introduction}

Tuberculosis (TB), remains a significant public health problem worldwide. Latent TB infection (LTBI) describes the condition wherein an individual is infected with Mycobacterium tuberculosis but not currently manifesting active disease (1). For a person with documented LTBI, the estimated lifetime risk of developing active TB is $10 \%$, although preventive treatment can avert this risk and confer an estimated protective effect of $60-90 \%(2,3)$. To reduce both the incidence of active TB and the associated screening costs, LTBI screening and treatment are applied selectively to those with high risk of developing active TB. However, target populations for screening vary among countries and guidelines $(1,4-7)$.

Patients with cancer may exhibit deficiencies in cellmediated immunity as either a direct effect or an indirect effect related to chemotherapy $(8,9)$. Accordingly, many patients with LTBI and cancer develop active TB $(10,11)$. However, the 2015 World Health Organization (WHO) guidelines do not recommend LTBI screening and treatment for patients with cancer, and the current Korean guidelines recommend targeting only patients with hematologic or head and neck cancers (7). Nonetheless, the WHO guidelines also emphasize the importance of further research on the benefits and harms of LTBI screening and treatment in this patient population (12).

Objectively quantifying the magnitude of active TB risk faced by patients with various malignancies remains challenging. Although a single-center study reported an increased incidence of TB in patients with cancer (9), no studies have investigated the risks associated with various malignancies in a geographical region. A recent meta-analysis by Cheng reported an increased risk of TB development in patients with solid cancers, particularly hematologic, head and neck, and lung cancers [incidence rate ratio $(I R R)=12]$. Accordingly, those patients faced a higher risk of developing active TB and would benefit from LTBI screening and treatment (13). Dobler et al. further performed a meta-analysis estimating the incidence of $\mathrm{TB}$ relative to a reference group after adjusting for age, and found that the IRR of TB associated with cancer was 2.61; those authors concluded that LTBI screening in patients with cancer may be unnecessary except for patients with hematologic malignancies and children with cancer (14).

The accuracy and reliability of TB diagnoses are often questionable, as many clinical diagnoses of TB are not confirmed using microbiological evidence such as cultures or polymerase chain reaction (PCR). Given the lack of studies investigating associations of active TB with multiple types of malignancies in a single population, as well as the conflicting results of previous studies, additional research is needed to better define high-risk patients and determine the optimal management of LTBI in patients with various malignancies. In this study, we aimed to evaluate the degree of risk of active TB, including bacteriologically confirmed TB (BCTB) and clinically diagnosed TB (CDTB), in patients with various cancers in Korea.

\section{Methods}

\section{Case cohort and control cohorts (control cohort 1 and control cobort 2)}

This study was approved by the Institutional Review Board of Ulsan University Hospital (no. UUH 2018-04-006). We retrospectively established case and control cohorts (control cohort 1 and control cohort 2) to estimate the relative risk of developing active TB. Patients aged $\geq 20$ years who were newly diagnosed with malignancies at Ulsan University Hospital (UUH) between January 2000 and December 2014 were collected as case cohort. Diagnoses of malignancy were based on International Classification of Diseases, Tenth Revision (ICD-10) codes (C000-C999). The malignancies and ICD-10 codes included in this study are listed in Table S1. In patients with multiple or duplicate cancers, the first registered cancer was selected for analysis. Patients who developed active TB within 2 years before a cancer diagnosis were excluded. Patients in whom active TB and cancer were diagnosed simultaneously were also excluded to avoid ascertainment bias. The development of active TB for 3 years after a cancer diagnosis was investigated in the case cohort (9).

Individuals who had no history of cancer, visited UUH for health screening during the case cohort enrollment period, and were followed for more than 3 years after health screening were enrolled randomly via a $1: 2$ sexand age-matching strategy as the control cohort 1 . Those who developed active TB within 2 years before enrollment were excluded. Those with active TB as determined by chest radiography or chest computed tomography (CT) at the time of enrollment were also excluded to avoid ascertainment bias. The development of active TB for 3 years after enrollment was also investigated in the control cohort 1 .

The incidence of newly developed ТВ was expressed 
in terms of 100,000 person-years (py) to account for differences in the follow-up periods between the case cohort and control 1 cohort. Clinical data of the case cohort and control cohort1 were extracted using the Ulsan University Hospital Information of Clinical Ecosystem, a clinical data warehouse.

We additionally set the total population of Ulsan province (Korea) as the control cohort 2 and surveyed the annual population of Ulsan from 2000-2017 and the number of TB patients per year for the period from the Korean Statistical Information Service (http://kosis.kr) to determine the incidence of TB.

\section{Definition of active tuberculosis}

Active TB was defined as follows: (I) the isolation of $M$. tuberculosis using any mycobacterial culture method; or (II) the isolation of $M$. tuberculosis using any molecular methods; or (III) a diagnosis of TB by a physician (ICD-10 codes A15-A19) AND prescription of medication for TB, such as isoniazid, rifampin (rifampicin), rifabutin, ethambutol, pyrazinamide, cycloserine, prothionamide, streptomycin, kanamycin, or amikacin. Patients who met criterion (I) or (II) were classified further as BCTB, while those who met criterion (III) were classified as CDTB. "All TB" included both BCTB and CDTB cases (15).

\section{Statistical analysis}

The IRR was used to estimate the risk of developing TB according in all malignancies and each type of malignancy were estimated using a Poisson regression analysis. The IRR was calculated by dividing the incidence rate (IR) of $\mathrm{TB}$ in the cancer cohort by that in the control cohorts; this was performed separately for each control cohort. All cancer patients were analyzed by age group, and additionally analyzed by cancer type in five most common malignancies of developing TB. All statistical analyses were performed using Stata SE software, version 12 (Stata Corporation, College Station, TX, USA) and R package, version 3.5.1 (R Project for Statistical Computing, Vienna, Austria).

\section{Results}

\section{Incidence rate of tuberculosis in the case cohort}

A total of 34,783 patients with cancer were enrolled, with a male proportion of $50.2 \%$ and median age of 58 years (interquartile range, 68-48). The numbers of cases of each type of cancer are shown in Table S2. During the study period, 496 of 34,783 cancer patients developed TB and the incidence rates of BCTB and all TB were 535 and $699 / 100,000$ py, respectively. The incidence rates of TB according to the type of cancer are shown in Table 1.

\section{Incidence rate of tuberculosis in the control cohorts 1 and 2}

As the cohort 1, 69,566 age- and sex-matched subjects were registered. The incidence rates of all TB and ВCTB in the control cohort 1 were 65 and 37/100,000 py, respectively.

In the control cohort 2 , the $\mathrm{TB}$ incidence rate was calculated from 2000 to 2017 using the annual changes in population and the number of TB patients per year. The estimated annual average population size was $1,151,402$ persons, and the average annual number of TB cases was 829. The calculated TB incidence rate in the control cohort 2 was 72/100,000 year; BCTB cases were not identified separately. The TB incidence rates in the control cohorts 1 and 2 are shown in Table 2.

\section{Incidence rate ratio of tuberculosis in the case cohort compared to the control cobort 1 and 2}

In the entire case cohort, the overall IRR for the development of all TB compared to the control cohort 1 was 10.68 [95\% confidence interval (CI), 8.83-12.99], while the IRR for BCTB was 14.30 (95\% CI, 11.91-17.18). Among all patients with cancer, the overall IRR for the development of all TB was 9.71 (95\% CI, 8.99-10.48), compared to control cohort 2. The IRRs for each type of cancer relative to the control cohorts 1 and 2 are shown in Table 3, Figures 1 and 2. Regardless of the type of control cohort, common cancers of developing all TB and BCTB include esophageal cancer, lung cancer, pancreatic cancer, head and neck cancer and hematologic malignancies. All cancer patients were analyzed by age group, and additionally analyzed by cancer type in five most common malignancies of developing TB, esophageal cancer, lung cancer, pancreatic cancer, head and neck cancer, and hematologic malignancies. The risk of developing TB increased with old age. The IRRs in overall patients and in each cancer types are shown in Table 4.

\section{Discussion}

This case-control cohort study revealed that patients with cancer at our institution faced a significantly increased risk 


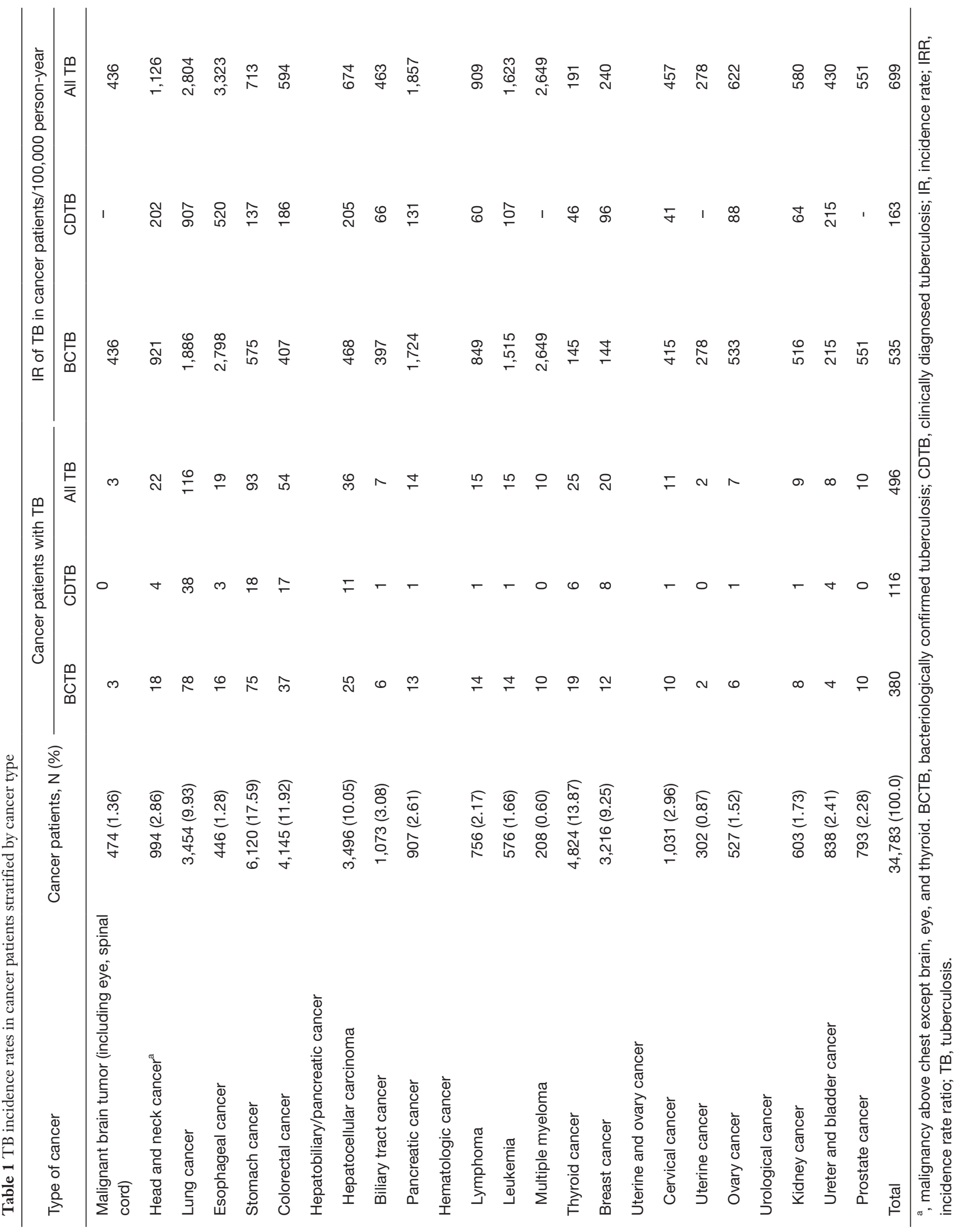


Table 2 Tuberculosis statistics in the control cohort 1 [1:2 matched (age/sex) health screening subjects] and control cohort 2 (total population of Ulsan province)

\begin{tabular}{lcccc}
\hline \multirow{2}{*}{ Parameters } & & Control 1 $(\mathrm{N}=69,566)$ & Control 2 & \\
\cline { 2 - 4 } & BCTB & CDTB & All TB & $\left(\mathrm{N}=1,151,402^{\mathrm{a}}\right)$ \\
\hline Number & 79 & 59 & 138 & $829^{\mathrm{a}}$ \\
Duration & $210,972.772$ person-year & $210,996.493$ person-year & $210,875.326$ person-year & 18 years \\
Incidence rate & $37 / 100,000$ person-year & 28/100,000 person-year & $65 / 100,000$ person-year & $72 / 100,000$ year \\
\hline
\end{tabular}

a, annual average. BCTB, bacteriologically confirmed tuberculosis; CDTB, clinically diagnosed tuberculosis.

Table 3 TB incidence rate ratios (IRRs) in cancer patients stratified by cancer types, compared to the control cohort 1 (age-/sex-matched health screening subjects, $1: 2$ ratio) and control cohort 2 (Ulsan population)

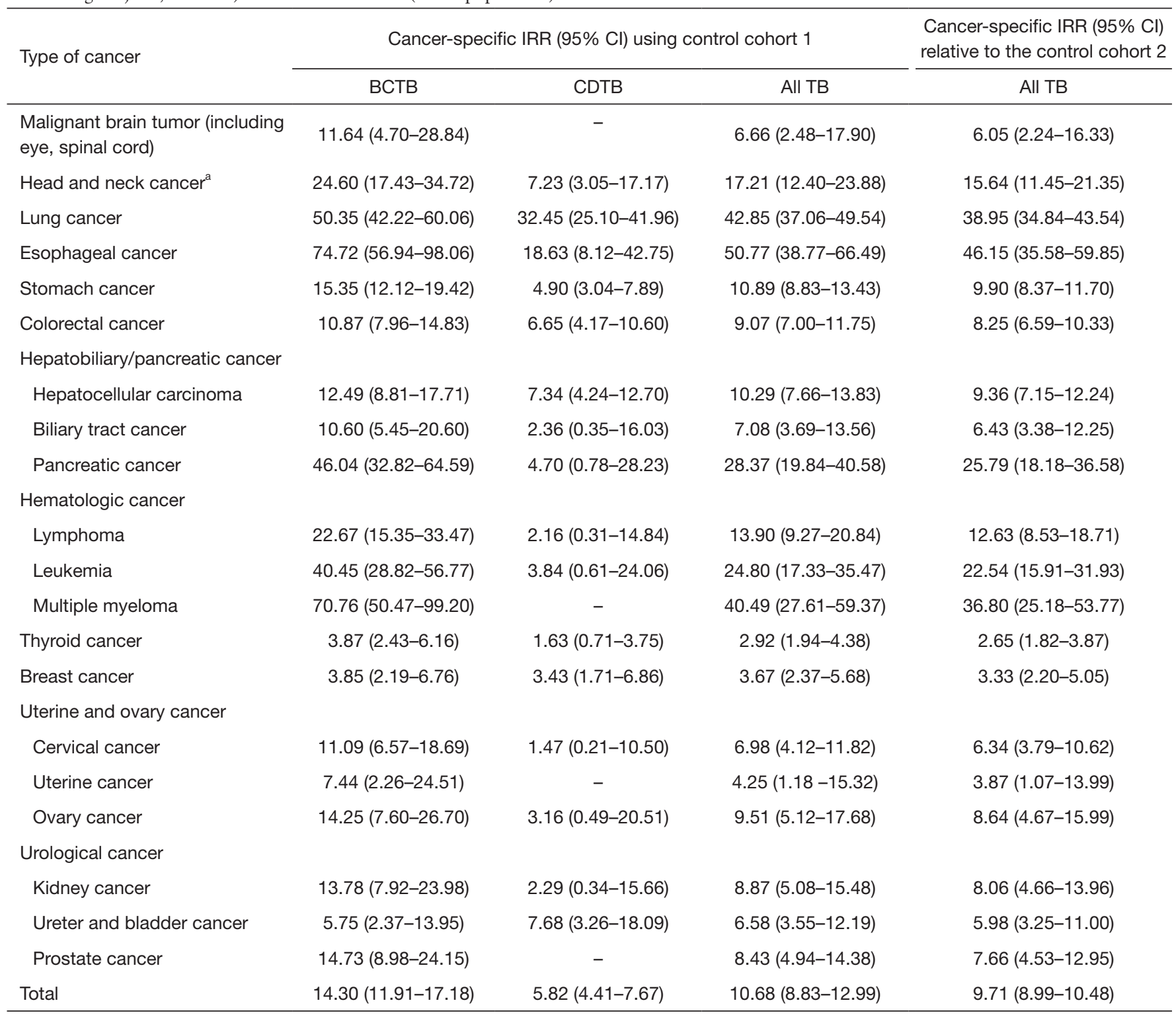

${ }^{\mathrm{a}}$, malignancy above the chest, except brain, eye, and thyroid. BCTB, bacteriologically confirmed tuberculosis; CDTB, clinically diagnosed tuberculosis; $\mathrm{Cl}$, confidence interval; IRR, incidence rate ratio; TB, tuberculosis. 
A

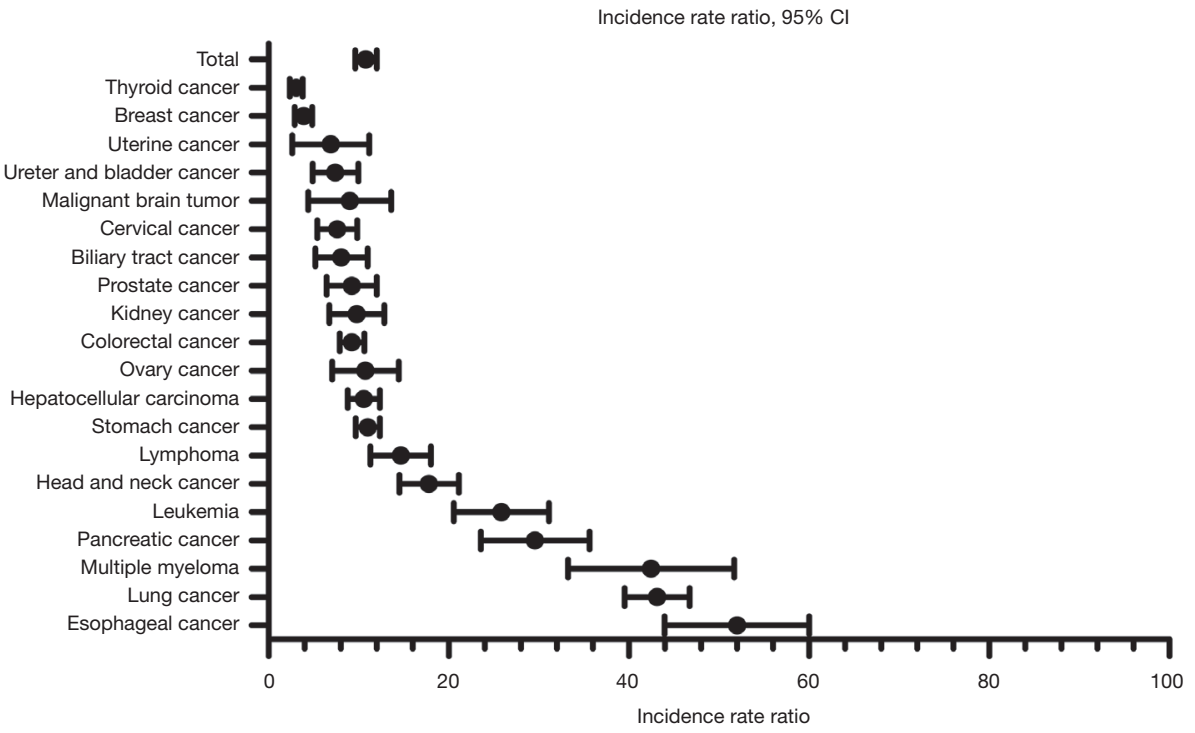

B

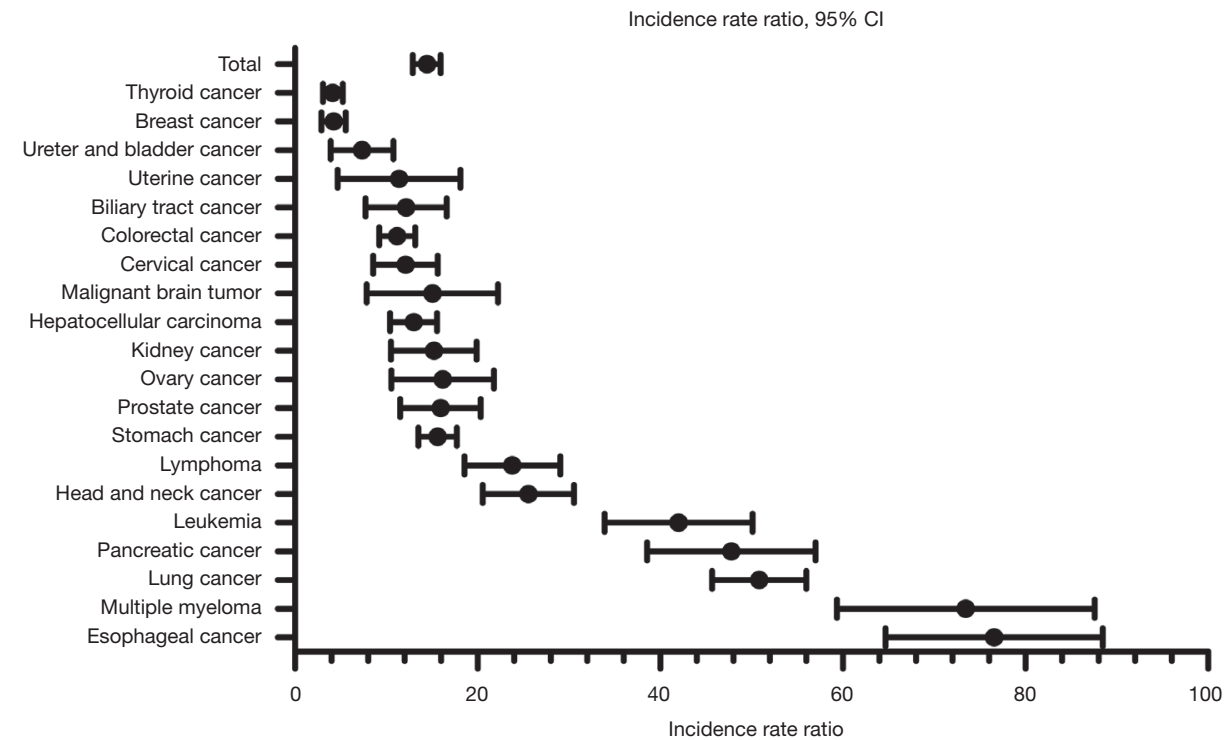

Figure 1 (A) Incidence rate ratios (IRRs) for all tuberculosis (TB) in cancer patients stratified by cancer types, compared to the control 1 cohort (health screening subjects); (B) IRRs for bacteriologically confirmed tuberculosis (BCTB) in cancer patients stratified by cancer types, compared to control 1 cohort (health screening subjects).

of developing TB relative to people who participated in a health screening program at the same institution or to the general population of Ulsan province. Moreover, IRRs were $\geq 20$-fold for the development of BCTB among patients with esophageal cancer, multiple myeloma, lung cancer, pancreatic cancer, leukemia, head and neck cancer, and lymphoma.

In contrast to previous studies, our study included almost all types of cancer commonly encountered in clinical practice, and our control cohort 1 included only subjects for whom detailed medical records were available for more than 3 years from the time of health screening. We further determined the TB incidence rate based on the total population of Ulsan province as the control cohort 2, as the control cohort 1 may not have been representative of the general population. Similar incidence rates of all TB were observed in the control cohorts 1 and 2, indicating that control 1 adequately represented the general population. Our findings, derived from a large sample extracted from a single regional population cohort to reduce heterogeneity, 


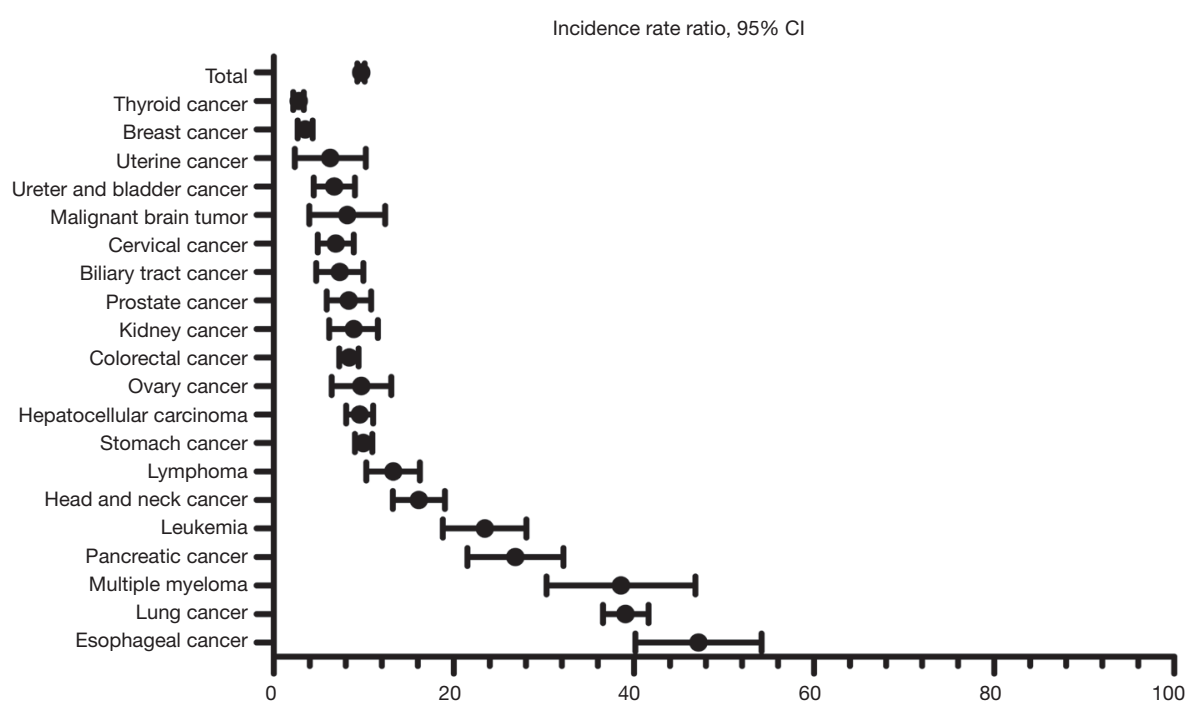

Figure 2 Incidence rate ratios (IRR) for all tuberculosis (TB) in cancer patients stratified by cancer types, compared to the control 2 cohort (population of Ulsan province).

provide a valuable and objective analysis of TB in patients with various malignancies. The IRRs of each malignancy were based on this single population cohort and are therefore more reliable than those obtained in previous meta-analyses involving various populations $(13,14)$. We also included only BCTB cases in some analyses to avoid diagnostic uncertainty (14).

We observed an approximately 10 -fold increase in the risk of developing TB among patients with cancer when compared with health screening participants and the general population of Ulsan. The IRRs of patients with cancer in our study were comparable to the estimated risks associated with the indications for current LTBI screening and treatment, such as AIDS (16), recent TB infection within 2 years (17), end-stage renal disease $(18,19)$, organ transplantation (20), and TNF-alpha inhibitor use (21).

Notably, we observed the highest relative risk of TB development among patients with esophageal cancer. This was consistent with a previous report by $\mathrm{Wu}$ et al., wherein the risk of $\mathrm{TB}$ was highest in patients with esophageal cancer but similar to the risks associated with hematologic malignancies and lung cancer (22). The increased risk of TB development in these patients may be confounded by the associations of heavy smoking and excessive alcohol use, which are also independent risk factors for active TB $(23,24)$. Despite these possible confounding factors, however, the relative risk of BCTB was significantly higher in patients with esophageal cancer than in the control group in our study.

In our study, patients with lung cancer also had a high IRR for TB and BCTB development, which was again consistent with a previous report (22). As the Korean guidelines for initial evaluation include pulmonary TB as a possible differential diagnosis of lung cancer, it may be difficult to obtain an accurate diagnosis of TB in patients with lung cancer. Therefore, a previous analysis by Seo et al. (25) excluded patients with lung cancer. Other studies have reported an increased risk of lung cancer in patients with a history of pulmonary TB $(26,27)$, thus obscuring the causal relationship between these diseases. Again, smoking may be a confounding factor for TB development in patients with lung cancer.

Hematologic malignancies are associated with an increased risk of developing active TB. In this study, although all patients with hematologic malignancies faced an increased risk, the risk of developing TB was particularly high among those with multiple myeloma, consistent with a previously reported incidence from a study in Taiwan (28). Compared to other hematologic malignancies, patients with multiple myeloma tend to be older and are more likely to develop chronic kidney disease and receive longterm steroid therapy and chemotherapy, which may account for the increased risk of TB (28). Therefore, previous recommendations regarding LTBI screening for patients with hematologic malignancies, especially multiple myeloma, should be upheld. 


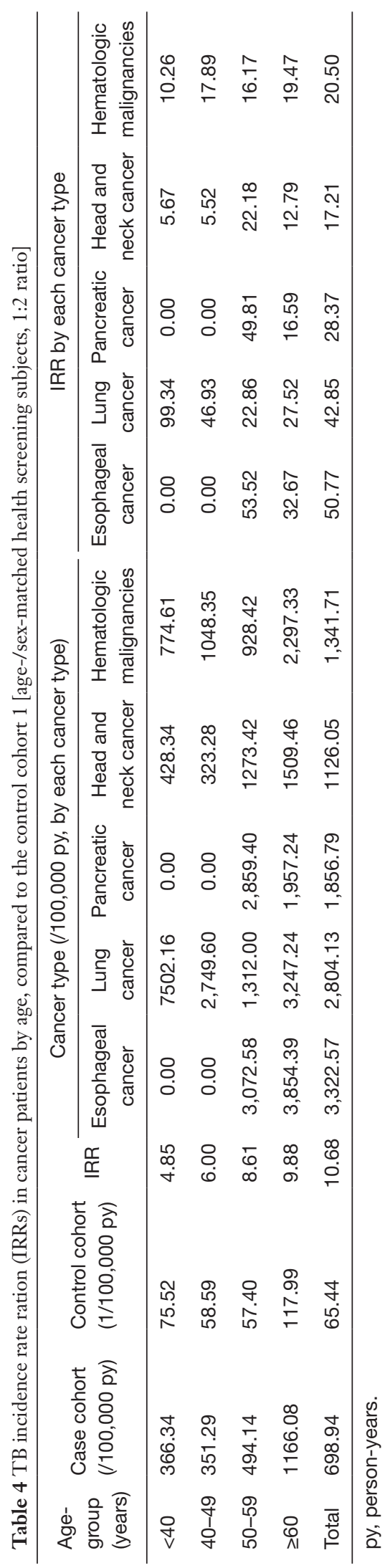

Previously, Seo et al. reported that pancreatic cancer was associated with the highest risk of TB among all solid organ cancers except lung cancer (25). In our study, pancreatic cancer was associated with the fourth highest risk of BCTB development among the investigated cancers. Most patients with pancreatic cancer are diagnosed at an advanced stage of disease, and the associated malnutrition, and deteriorated general condition might be associated with a higher risk of TB development. Moreover, patients with advanced pancreatic cancer have a markedly reduced life expectancy, which is also associated with an increased IRR for active TB. Still, pancreatic cancer has a much higher IRR for TB bile duct or liver cancer, although both latter cancers are also largely diagnosed at an advanced stage and are associated with a short life expectancy. Accordingly, currently unknown and potentially independent mechanisms may contribute to the development of TB in patients with pancreatic cancer, such as tumor-associated inflammation, the decreased production of immune-related proteins due to decreased fat absorption, and cancer-induced diabetes. Further research is needed to clarify this mechanism.

A diagnosis of active TB affects the treatment administered to a cancer patient. For example, chemotherapy or surgery may be delayed due to a TB diagnosis or associated medication therapy. In a study by Ahn et al., the identification of TB infection interrupted the administration of bortezomib in patients with multiple myeloma, which significantly affected patient outcomes (29). LTBI screening at the time of a diagnosis of a high TB risk cancer may enable the prevention of active TB during cancer treatment and enable the appropriate administration of chemotherapy.

Developments in chemotherapeutic drugs and treatment modalities for cancer patients have led to increases in life expectancies. It is increasingly important to identify patients at a high risk of developing active TB and provide appropriate treatment. As noted previously, cancer patients with decreased cellular immunity face an increased risk of active TB (30) and remain in a prolonged state of immunocompromise. Therefore, LTBI screening and treatment is needed to prevent opportunistic infection and should be performed at the time of cancer diagnosis, as LTBI screening is performed at the time of HIV diagnosis in developing countries.

This study had several limitations. First, it was performed retrospectively at a single center, and therefore the results may not reflect the general population in Korea. Further, risk of active tuberculosis in cancer patients depends to great extent on tuberculosis epidemiological situation in 
certain geographic region. Therefore, the results of this study should be carefully generalized in countries with low TB incidence. Second, the possibility of sampling bias in the diagnosis of active TB could not be excluded. Patients with malignancy usually received more medical attention in terms of frequent physical examination, or medical procedure such as laboratory or radiologic tests. The third shortcoming was that we could not identify comorbidities such as diabetes, malnutrition, HIV infection, renal insufficiency and immunosuppressant therapies that might have affected the development of TB. These potential predisposing factors were not extracted because the data of comorbidities were not accurately collected from big data. Other factors such as the disease stage at cancer diagnosis, chemotherapy, surgery, and radiotherapy, were not included in the analysis. Lastly, our study does not justify unconditional LTBI testing for cancers with high TB incidence. If there are a previous history of tuberculosis treatment and a radiologically stable scar lesion, LTBI testing is not necessary. In addition, if there is currently evidence of active tuberculosis, tuberculosis treatment may be performed without the LTBI test. The indications for LTBI and preventive anti-tuberculous therapy in cancer patients should be much better defined by future studies.

In conclusion, the incidence of active TB was higher in cancer patients than in health screening recipients or the general population of Ulsan province. Patients with lung cancer, esophageal cancer, pancreatic cancer, hematologic malignancy, and head and neck cancer faced a particularly high risk of developing TB. Therefore, we recommend targeted LTBI screening and treatment strategies for patients with these high-risk cancers.

\section{Acknowledgments}

Funding: This work was supported by grants from Ulsan University Hospital [Biomedical Research Center Promotion Fund, UUH-2018-07] and the National Research Foundation of Korea [NRF-2017R1C1B5076493].

\section{Footnote}

Conflicts of Interest: All authors have completed the ICMJE uniform disclosure form (available at http://dx.doi. org/10.21037/jtd.2020.02.50). The authors have no conflicts of interest to declare.
Ethical Statement: The authors are accountable for all aspects of the work in ensuring that questions related to the accuracy or integrity of any part of the work are appropriately investigated and resolved. This study was approved by the Institutional Review Board of UUH (approval no. 2018-04-006) and was performed in accordance with the ethical standards of the institutional research committee and the latest Declaration of Helsinki. Informed consent was waived for its nature of retrospective analysis.

Open Access Statement: This is an Open Access article distributed in accordance with the Creative Commons Attribution-NonCommercial-NoDerivs 4.0 International License (CC BY-NC-ND 4.0), which permits the noncommercial replication and distribution of the article with the strict proviso that no changes or edits are made and the original work is properly cited (including links to both the formal publication through the relevant DOI and the license). See: https://creativecommons.org/licenses/by-nc-nd/4.0/.

\section{References}

1. Taylor Z, Nolan CM, Blumberg HM. Controlling tuberculosis in the United States: recommendations from the American thoracic society, CDC, and the Infectious Diseases Society of America. MMWR Recomm Rep 2005;54:1-81.

2. Snider DE Jr, Caras GJ, Koplan JP. Preventive therapy with isoniazid: cost-effectiveness of different durations of therapy. JAMA 1986;255:1579-83.

3. Lecoeur HF, Truffot-Pernot C, Grosset JH. Experimental short-course preventive therapy of tuberculosis with rifampin and pyrazinamide. Am Rev Respir Dis 1989;140:1189-93.

4. Tuberculosis: NICE guideline 2016. Available online: https://www.nice.org.uk/guidance/ng33

5. Canadian Tuberculosis Standards. 7th ed. Ottawa: Public Health Agency of Canada; 2014.

6. Guidelines on the management of latent tuberculosis infection. Geneva: World Health Organization; 2015.

7. Joint Committee for the Revision of Korean Guidelines for Tuberculosis. Korean guidelines for tuberculosis. 3rd ed. Cheongju: Korea Centers for Disease Control and Prevention; 2017.

8. O'Garra A, Redford PS, McNab FW, et al. The immune response in tuberculosis. Annual review of immunology. 
2013;31:475-527.

9. Kim HR, Hwang SS, Ro YK, Jeon CH, Ha DY, Park SJ, et al. Solid-organ malignancy as a risk factor for tuberculosis. Respirology 2008;13:413-9.

10. De La Rosa GR, Jacobson KL, Rolston KV, Raad II, Kontoyiannis DP, Safdar A. Mycobacterium tuberculosis at a comprehensive cancer centre: active disease in patients with underlying malignancy during 1990-2000. Clin Microbiol Infect 2004;10:749-52.

11. Kamboj M, Sepkowitz KA. The risk of tuberculosis in patients with cancer. Clin Infect Dis 2006;42:1592-5.

12. Getahun H, Matteelli A, Abubakar I, et al. Management of latent Mycobacterium tuberculosis infection: WHO guidelines for low tuberculosis burden countries. The European respiratory journal. 2015;46:1563-76.

13. Cheng MP, Abou Chakra CN, Yansouni CP, et al. Risk of Active Tuberculosis in Patients with Cancer: A Systematic Review and Meta-Analysis. Clinical infectious diseases : an official publication of the Infectious Diseases Society of America. 2017;64(5):635-44.

14. Dobler CC, Cheung K, Nguyen J, et al. Risk of tuberculosis in patients with solid cancers and haematological malignancies: a systematic review and meta-analysis. Eur Respir J 2017;50:1700157.

15. Kim CH, Im KH, Yoo SS, et al. Comparison of the incidence between tuberculosis and nontuberculous mycobacterial disease after gastrectomy. Infection. 2014;42:697-704.

16. Guelar A, Gatell JM, Verdejo J, et al. A prospective study of the risk of tuberculosis among HIV-infected patients. AIDS 1993;7:1345-9.

17. Sutherland I. Recent studies in the epidemiology of tuberculosis, based on the risk of being infected with tubercle bacilli. Adv Tuberc Res 1976;19:1-63.

18. Hussein MM, Mooij JM, Roujouleh H. Tuberculosis and chronic renal disease. Semin Dial 2003;16:38-44.

19. Christopoulos AI, Diamantopoulos AA, Dimopoulos PA, et al. Risk factors for tuberculosis in dialysis patients: a prospective multi-center clinical trial. BMC Nephrol

Cite this article as: Cheon J, Kim C, Park EJ, Ock M, Lee H, Ahn JJ, Jegal Y, Seo KW, Park SE, Han I, Kang H, An M, Park J, Park GM, Jun JB, Lee T. Active tuberculosis risk associated with malignancies: an 18-year retrospective cohort study in Korea. J Thorac Dis 2020;12(9):4950-4959. doi: 10.21037/jtd.2020.02.50
2009;10:36.

20. Torre-Cisneros J, Doblas A, Aguado JM, et al. Tuberculosis after solid-organ transplant: incidence, risk factors, and clinical characteristics in the RESITRA (Spanish Network of Infection in Transplantation) cohort. Clin Infect Dis 2009;48:1657-65.

21. Brassard P, Kezouh A, Suissa S. Antirheumatic drugs and the risk of tuberculosis. Clin Infect Dis 2006;43:717-22.

22. Wu CY, Hu HY, Pu CY, et al. Aerodigestive tract, lung and haematological cancers are risk factors for tuberculosis: an 8-year population-based study. Int J Tuberc Lung Dis 2011;15:125-30.

23. Alavi-Naini R, Sharifi-Mood B, Metanat M. Association between tuberculosis and smoking. Int J High Risk Behav Addict 2012;1:71-4.

24. Volkmann T, Moonan PK, Miramontes R, Oeltmann JE. Tuberculosis and excess alcohol use in the United States, 1997-2012. Int J Tuberc Lung Dis 2015;19:111-9.

25. Seo GH, Kim MJ, Seo S, et al. Cancer-specific incidence rates of tuberculosis: A 5-year nationwide populationbased study in a country with an intermediate tuberculosis burden. Medicine 2016;95:e4919.

26. Wu CY, Hu HY, Pu CY, et al. Pulmonary tuberculosis increases the risk of lung cancer: a population-based cohort study. Cancer 2011;117:618-24.

27. Liang HY, Li XL, Yu XS, et al. Facts and fiction of the relationship between preexisting tuberculosis and lung cancer risk: a systematic review. International journal of cancer. 2009;125:2936-44.

28. Tsai CK, Huon LK, Ou SM, et al. Risk and impact of tuberculosis in patients with multiple myeloma. Leuk Lymphoma 2017;58:2598-606.

29. Ahn JS, Rew SY, Yang DH, et al. Poor prognostic significance of Mycobacterium tuberculosis infection during bortezomib-containing chemotherapy in patients with multiple myeloma. Blood Res 2013;48:35-9.

30. Safdar A, Armstrong D. Infectious morbidity in critically ill patients with cancer. Critical Care Clinics 2001;17:53170 , vii-viii. 
Supplementary

Table S1 Malignancies according to ICD-10 codes

\begin{tabular}{|c|c|}
\hline Type of cancer & ICD-10 codes \\
\hline $\begin{array}{l}\text { Malignant brain tumor (including } \\
\text { eye, spinal cord) }\end{array}$ & C69-C72 \\
\hline Head and neck cancer ${ }^{a}$ & $\mathrm{C} 00-\mathrm{C} 14, \mathrm{C} 30-\mathrm{C} 32, \mathrm{C} 76$ \\
\hline Lung cancer & C33-C34 \\
\hline Esophageal cancer & C15 \\
\hline Stomach cancer & C16 \\
\hline Colorectal cancer & C18-C20 \\
\hline \multicolumn{2}{|l|}{ Hepatobiliary/pancreatic cancer } \\
\hline Hepatocellular carcinoma & $\mathrm{C} 22$ \\
\hline Biliary tract cancer & C23-C24 \\
\hline Pancreatic cancer & $\mathrm{C} 25$ \\
\hline Thyroid cancer & $\mathrm{C} 73$ \\
\hline Breast cancer & C50 \\
\hline \multicolumn{2}{|l|}{ Uterine and ovary cancer } \\
\hline Cervical cancer & C53 \\
\hline Uterine cancer & C54-C55 \\
\hline Ovary cancer & C56 \\
\hline \multicolumn{2}{|l|}{ Urological cancer } \\
\hline Kidney cancer & C53 \\
\hline Ureter and Bladder cancer & C54-C55 \\
\hline Prostate cancer & C56 \\
\hline \multicolumn{2}{|l|}{ Hematologic cancer } \\
\hline Lymphoma & C81-C88 \\
\hline Leukemia & C91-C95 \\
\hline Multiple myeloma & $\mathrm{C90}$ \\
\hline
\end{tabular}

a , malignancy above the chest, except brain, eye, and thyroid. 


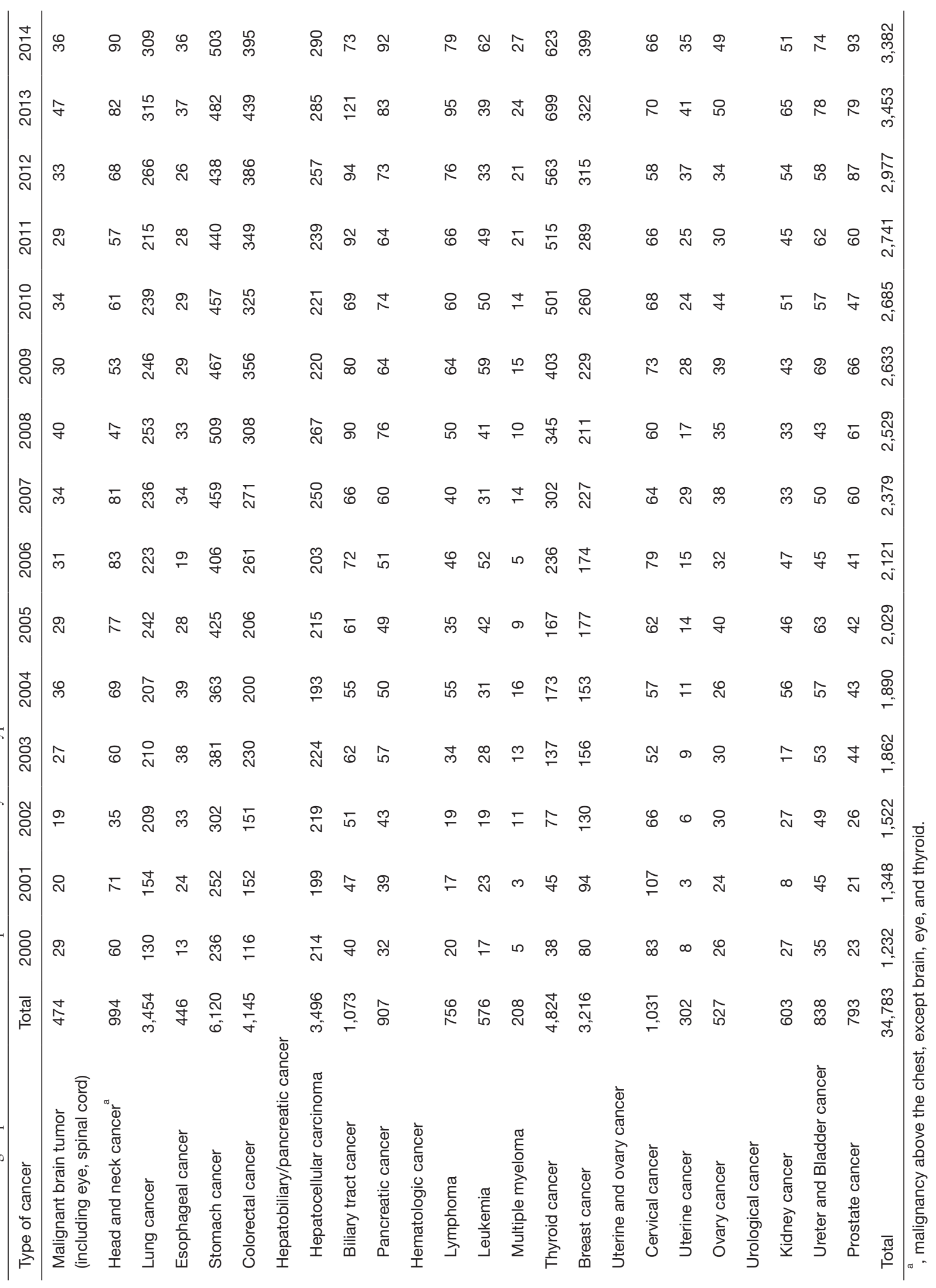

